\title{
Dem PMT-Gesetz fehlt die Verfassungsgrundlage
}

Für das vom Eidgenössischen Parlament am 25. September 2020 beschlossene Gesetz zur präventiven Terrorismusbekämpfung fehlt dem Bund die verfassungsmässige Kompetenz. Keine der vom Bund angeführten Begründungen seiner Zuständigkeit halten einer Prüfung stand. Der Erlass polizeigesetzlicher Bestimmungen zur Verhütung von Straftaten durch operationelle Realakte als Eingriffe in die Bewegungsfreiheit liegt in der Verantwortung der Kantone. Dies gilt auch im Bereich der präventiven Terrorismusbekämpfung als Teil des Staatsschutzes. Dieser an sich unbefriedigende Rechtszustand lässt sich nur durch eine Änderung der Bundesverfassung beheben. In einem folgenden Beitrag werden die polizeilichen Massnahmen zur Bekämpfung von Terrorismus (PMT) in Bezug auf ihre Verfassungs- und EMRKKonformität sowie ihre Wirksamkeit beurteilt.

I. Vorbemerkung und Gliederung

II. Zur Gesetzgebungskompetenz des Bundes: Die geltende Kompetenzregelung $\ldots \ldots \ldots \ldots \ldots \ldots \ldots \ldots$

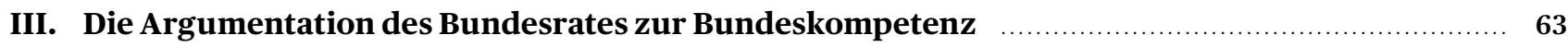

1. Zur Prävention in der Terrorismusbekämpfung $\ldots \ldots \ldots \ldots \ldots \ldots \ldots \ldots \ldots \ldots \ldots \ldots \ldots \ldots \ldots \ldots \ldots \ldots \ldots$

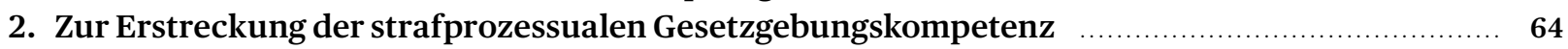

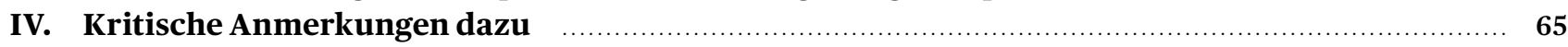

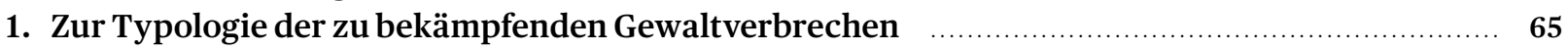

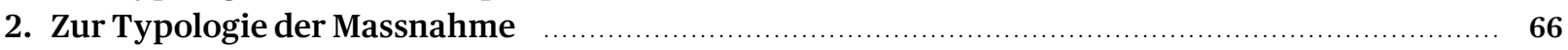

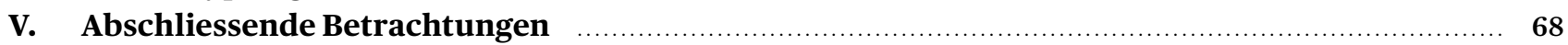

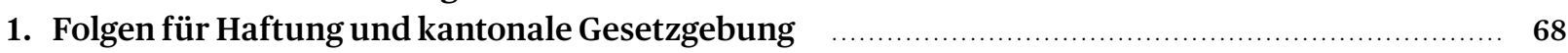

2. Zum Fehlen einer effektiven Verfassungsgerichtsbarkeit gegenüber Bundesgesetzen $\quad \ldots \ldots \ldots \ldots \ldots$

3. Das PMT-Gesetz ist kein Einzelfall: Die wiederholte Missachtung der bundesstaatlichen Zuständigkeitsordnung im Sicherheits- und Polizeirecht $\quad \ldots \ldots \ldots \ldots \ldots \ldots \ldots$

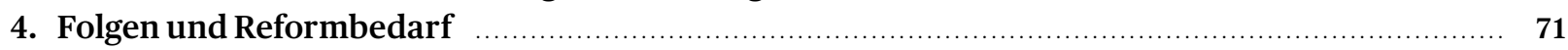

Zitiervorschlag:

MARKUS MOHLER, Dem PMT-Gesetz fehlt

die Verfassungsgrundlage, sui generis 2021, S. 61 


\section{Vorbemerkung und Gliederung}

1 Am 25. September 2020 haben die eidgenössischen Räte als vorläufigen Umsetzungs-Abschluss der Strategie der Schweiz zur Terrorismusbekämpfung1 das Bundesgesetz über polizeiliche Massnahmen zur Bekämpfung von Terrorismus (PMT) verabschiedet. ${ }^{2}$ Es handelt sich um ein Mantelgesetz ${ }^{3}$ durch das hauptsächlich das Bundesgesetz über Massnahmen zur Wahrung der inneren Sicherheit vom 21. März 1997 (BWIS)4 ${ }^{4}$ und zudem zwölf weitere Bundesgesetze ${ }^{5}$ teilrevidiert werden.

2 In verschiedener Hinsicht wirft dieser Erlass gewichtige Fragen auf. Es handelt sich, wie es der Name des Gesetzes sagt, soweit das BWIS dadurch novelliert wird, um Massnahmen im sicherheitspolizeilichen Bereich ${ }^{6}$ Es geht um die Verhinderung terroristischer Aktivitäten als vorbeugende Massnahmen, ${ }^{7}$ demnach um Prävention in einer ihrer Ausprägungen. ${ }^{8}$

3 Aus Platzgründen werden diese Ausführungen zu den Änderungen des BWIS in zwei Teilen dargelegt. Der vorliegende erste Teil widmet sich der Frage nach der Bundeskompetenz zum Erlass dieses Gesetzes, soweit es die Änderung des BWIS betrifft. Sie hat in der parlamentarischen und rechtswissenschaftlichen Debatte nicht die gebührende Beachtung gefunden (II-IV). Danach folgen weitergehende Überlegungen zum Spannungsverhältnis zwischen Föderalismus und Sicherheits- bzw. Polizeirechtsgesetzgebung, also zu einem grundlegenden Problem des Staatsorganisationsrechts (V).

4 Der zweite, später folgende Teil wird die Begriffe «terroristischer Gefährder» bzw. «terroristische Aktivitäten» und die damit verbundene grundrechtliche Problematik sowie die einzelnen Massnahmen und deren Voraussetzungen behandeln.

1 Strategie der Schweiz zur Terrorismusbekämpfung vom 18. September 2015 (BBl 20157487).

2 Bundesgesetz vom 25. September 2020 über polizeiliche Massnahmen zur Bekämpfung von Terrorismus (PMT) (BBl 2020 7741).

3 Botschaft vom 22. Mai 2019 zum Bundesgesetz über polizeiliche Massnahmen zur Bekämpfung von Terrorismus (BBl 2019 4751), S. $4846 \mathrm{f}$.

4 Bundesgesetz über Massnahmen zur Wahrung der inneren Sicherheit vom 21. März 1997 (BWIS; SR120).

5 Vgl. Botschaft zum Bundesgesetz über polizeiliche Massnahmen zur Bekämpfung von Terrorismus (Fn.3), S.4860 ff.

6 So auch SVEn ZIMmERLIN, Das Bundesgesetz über polizeiliche Massnahmen zur Bekämpfung des Terrorismus (PMT), Sicherheit \& Recht $3 / 2020$, S. 189 .

7 Art. 2 Abs. 2 Bst. d ${ }^{\text {bis }}$ BWIS (neu).

8 Siehe dazu MARKUS H.F. MOHLER, Polizeiberuf und Polizeirecht im Rechtsstaat, 2020, S. $56 \mathrm{f}$.

\section{Zur Gesetzgebungskompetenz des Bundes: Die geltende Kompetenzregelung}

Dem Bund kommen nach Art. 3 i.V.m Art. 42 Abs. 1 BV nur 5 Kompetenzen zu, die ihm durch die Bundesverfassung 9 als konkrete Einzelermächtigungen zugewiesen worden sind. ${ }^{10}$ Vorbehalten bleiben nur die wenigen ungeschriebenen bzw. stillschweigenden ${ }^{11}$ inhärenten Kompetenzen. Solche bestehen nur punktuell für sicherheitspolizeiliche Vorkehrungen zum Schutz seiner eigenen Institutionen sowie völkerrechtlicher Schutzverpflichtungen. ${ }^{2}$ Die ebenso geringen impliziten Gesetzgebungsbefugnisse qua engen Sachzusammenhang fallen in diesem Bereich nicht in Betracht. Es liegt kein Sachzusammenhang mit einer originären, expliziten Bundeskompetenz vor, der eine adhäsionsweise Befugnis unausweichlich erscheinen lässt, damit die eigentliche Sachkompetenz überhaupt wahrgenommen werden kann bzw. ohne die deren Umsetzung unmöglich wäre. ${ }^{13}$

In der Terrorismusbekämpfung als Teil des Staatsschutzes 6 beschränkt sich der präventive Teil polizeirechtlicher Massnahmen auf die Informationsbeschaffung. ${ }^{14}$ Dies ist

9 Bundesverfassung der Schweizerischen Eidgenossenschaft vom 18. April 1999 (BV; SR 101).

10 Statt vieler: GIOVANNI BIAGGINI, in: Waldmann/Belser/Epiney (Hrsg.), Basler Kommentar, Bundesverfassung (BV), Basel 2015, Art. 3 N6, Art. 42 N1 (zit. BSK BV-BEARBEITERIN); TOBIAS JAAG, Kompetenzverteilung zwischen Bund und Kantonen, in: Biaggini/ Gächter/Kiener (Hrsg.), Staatsrecht, 2. Aufl.,Zürich/St. Gallen 2015, $\$ 12$ N 3; ULRICH HÄFELIN / WALTER HALLER / HELEN KELLER / DANIELA THURNHERR, Schweizerisches Bundesstaatsrecht, 10. Aufl., Zürich 2020 (zit. HÄFELIN et al.), N 1053; RENÉ RHINOW / MARKUS SCHEFER / PETER UEBERSAX, Schweizerisches Verfassungsrecht, 4. Aufl., Basel 2016, N694ff.; RAINER J. SCHWEIZER, in: Ehrenzeller/ Schindler/Schweizer/Vallender (Hrsg.), St. Galler Kommentar zur Bundesverfassung, 3. Aufl., Zürich/St. Gallen 2014, Art. 3 N10 m.w.N. (zit. SGK BV-BEARBEITERIN); JÜRG MARCEL TIEFENTHAL, Kantonales Polizeirecht der Schweiz, Zürich 2018, \$1 N1; PIERRE TSCHANNEN, Staatsrecht der Schweizerischen Eidgenossenschaft, 4. Aufl., Bern 2016, §19 N4.

11 Vgl. MARKUS H.F. MOHLER, Föderalismus im Sicherheits- und Polizeirecht - Reform dringend!, Newsletter IFF (Institut für Föderalismus der Universität Freiburg), 1/2018, S. 7 m.w.H.; TSCHANNEN, Staatsrecht (Fn.10), \$20 N12.

12 GIOVANNI BIAGGINI, Bundesverfassung der Schweizerischen Eidgenossenschaft, 2. Aufl., Zürich 2017, Art. 57 N6 (zit. OFK BV-BEARBEITERIN); BSK BV-DIGGELMANN/ALTWICKER, Art. 57 N 38; SGK BVSCHWEIZER/MOHLER, Vorbem. zur Sicherheitsverfassung N 26. Kritisch dazu noch PETER SALADIN, in: Aubert et al. (Hrsg.), Kommentar zur aBV, Basel et al. Stand 1986, Art. 3 N132.

13 CHRISTIAN LINSI, Verfassungsrechtliche Zuständigkeit für den Erlass von Bundesrecht, LeGes 2008/3, S. 475, mit Verweis auf die Entstehungsgeschichte; MOHLER, Föderalismus (Fn.11), S. 9; MARKUS H.F. MOHLER / RAINER J. SCHWEIZER, Sicherheitspolitik und Sicherheitsrecht - sicherheitsrechtliche Problemstellungen im Zusammenhang mit dem sicherheitspolitischen Bericht, Jusletter vom 7. Dezember 2009, N29; PIERRE TSCHANNEN, Staatsrecht (Fn.10), $\$ 20 \mathrm{~N} 12 \mathrm{f}$.

14 Art. 6 Abs.1 lit. a,Ziff.1 des Bundesgesetzes vom 25. September 2015 über den Nachrichtendienst(NDG; SR 121). Bericht des Bundesrates in Erfüllung des Postulats Malama 10.3045 vom 3. März 2010 
die hauptsächliche Aufgabe des Nachrichtendienstes des Bundes in enger Zusammenarbeit mit den entsprechenden kantonalen Diensten. ${ }^{15}$ Die gesetzliche Grundlage dafür liefert das Bundesgesetz über den Nachrichtendienst. ${ }^{16}$ Sicherheitspolizeiliche Massnahmen, d.h. operationelle Realakte wie vorläufige Festnahmen, gehören nicht dazu.

7 Aus Art. 42 BV fliesst, dass die Bundeskompetenzen $a b$ schliessend aufgezählt sind. Umgekehrt begründet Art. 3 BV die subsidiäre Generalkompetenz der Kantone: Sie üben alle Rechte aus, die dem Bund nicht übertragen sind. ${ }^{17}$ Ergänzt wird diese durch das Subsidiaritätsprinzip von Art. $5 a$ BV. ${ }^{18}$ Danach soll der Bund nicht Aufgaben übernehmen, welche die Kantone zumindest ebenso gut erfüllen können.19

8 Daraus ergibt sich, dass eine Zuständigkeitsübertragung bloss gestützt auf ein Bundesgesetz, eine (vermeintliche) Lückenfüllung oder Gewohnheitsrecht untersagt ist. ${ }^{20}$

9 Dem BundisteinesicherheitspolizeilicheZuständigkeit, also eine entsprechende Verbandskompetenz, explizit nicht übertragen worden. In der BV kommt der Ausdruck «Polizei» auch nicht vor. Sodann begründet Art. 57 BV nach übereinstimmender Lehre keine Bundeskompetenz. ${ }^{21}$

Innere Sicherheit. Klärung der Kompetenzen, vom 2. März 2012 (BBl 2012 4459), S. 4499; GABRIEL GERTSCH / ARMIN STÄHLI, Nachrichtendienstlicher Schutz, in: Kiener/Bühler/Schindler (Hrsg.), Sicherheits- und Ordnungsrecht des Bundes, Bd. III, Teil 2, Basel 2018, N30; REGINA KIENER / ANDREAS LEUZINGER, Verdeckte polizeiliche Massnahmen, in: Kiener/Bühler/Schindler (Hrsg.), Sicherheits- und Ordnungsrecht des Bundes, Bd. III, Teil 2, Basel 2018, N9.

15 Vgl. Art. 4 Abs. 1BWIS («Für die innere Sicherheit seines Gebietes ist in erster Linie der Kanton verantwortlich.»), Art. 4 lit. a NDG.

16 Art. 6 Abs. 1 lit. a Ziff. 1 NDG («Die Informationsbeschaffung und-bearbeitung des NDB dient: a. dem frühzeitigen Erkennen und Ver hindern von Bedrohungen der inneren oder äusseren Sicherheit, die ausgehen von: 1 . Terrorismus...»; Hervorhebung hier).

17 BSK BV-BIAGGINI, Art. 3 N6; JAAG (Fn.10), N3; RHINOW/SCHEFER/ UEBERSAX (Fn.10), N 706 ff.; SGK BV-sCHWEIZER, Art. 3 N 10; TSCHANNEN, Staatsrecht (Fn.10), §19 N 9.

18 Betr. Art. $43 a$ BV siehe nachfolgend Rz.15, 38.

19 OFK BV-BIAGgINI, Art. $5 a$ N 1, 6 ff.; ANDREAS GLASER, in: Biaggini/ Gächter/Kiener (Hrsg.), Staatsrecht, 2. Aufl., Zürich/St. Gallen 2015, §2 N72, S. 82; ANDREAS KLEY, in: Biaggini/Gächter/Kiener (Fn. 10), \$10 N20 f.; RAINER J. SCHWEIZER, in: Diggelmann/Randall/Schindler (Hrsg.), Verfassungsrecht der Schweiz, Bd.1, Zürich 2020, III.1 N 7, III.7 N7.

20 OFK BV-BIAGgINI, N 8 zu Art. 3; BSK BV-BIAGGINI, Art. 3 N 45; RHINOW/SCHEFER/UEBERSAX (Fn.10), N719; SGK BV-SCHWEIZER, Art. 3 N12; TSCHANNEN, Staatsrecht (Fn.10), §19 N 5 .

21 Vgl. etwa OFK BV-BIAGgINI, Art. 57 N2, 4; RETO PATRICK MÜLLER, Innere Sicherheit der Schweiz, Diss. Basel, Einsiedeln 2009, S. 419f.; SCHWEIZER (Fn. 19), S. 694; SGK BV-SCHWEIZER/MOHLER, Vorbem. Sicherheitsverfassung N 37; JÜRG MARCEL TIEFENTHAL, Kantonale Polizeihoheit, Zürich 2016, S. 14 f.; ZIMMERLIN (Fn. 6), S. 189; so auch im Vorentwurf und erläuternder Bericht zur Eröffnung des Vernehmlassungsverfahrens (BBl 2017 7875) zum Bundesgesetz über polizeiliche Massnahmen zur Bekämpfung von Terrorismus (PMT) vom 19. Dezember 2017 (zit. Erl. Ber. PMT).
Der Erlass sicherheitspolizeilicher Bestimmungen fällt somit, mit dem Vorbehalt des Selbstschutzes und völkerrechtlicher Schutzverpflichtungen (vorstehend Rz. 5), in die Zuständigkeit der Kantone, in deren Polizeihoheit. ${ }^{22}$

\section{Die Argumentation des Bundesra- tes zur Bundeskompetenz}

Der Bundesrat begründet die Kompetenz des Bundes 10 zum Erlass sicherheitspolizeilicher Bestimmungen mit zwei unterschiedlichen Argumentationslinien. Nach der ersten solle eine Lücke in der Bundesgesetzgebung gefüllt werden, wozu der Bund qua inhärenter Zuständigkeit die Kompetenz habe. Die zweite folgt einer neuen Auffassung, wonach der Bund gestützt auf die Zuständigkeit zur Gesetzgebung auf dem Gebiet des Strafprozessrechts die diesem quasi vorgelagerten polizeirechtlich nötigen Massnahmen festlegen dürfe.

\section{Zur Prävention in der Terrorismusbekämpfung}

Zunächst wird dargetan, der Bundesrat habe aufgrund 11 einer Analyse des geltenden bundesrechtlichen Instrumentariums die Lücken identifiziert, die im Massnahmendispositiv zu schliessen seien.23

Der Bundesrat begründet sodann die Verfassungskon- 12 formität zunächst mit der inhärenten Bundeskompetenz zur Wahrung der inneren und äusseren Sicherheit der Schweiz. Dies war im bisherigen Ingress des BWIS so aufgeführt: «die Zuständigkeit des Bundes zur Wahrung der inneren und äusseren Sicherheit der Eidgenossenschaft», worauf nun aber verzichtet werden soll. Stattdessen werde Art. 173 Abs. 2 BV angeführt, worin diese Regelungskompetenz enthalten sei. ${ }^{24}$ Der Bund habe «den Bestand des schweizerischen Gemeinwesens zu gewährleisten und für die Abwehr von Gefahren zu sorgen, die dieses existenziell bedrohen». Es handle sich um «Kompetenzen, die sich aus der staatlichen Existenz der Eidgenossenschaft ergeben». «Die von terroristischen Aktivitäten ausgehenden Gefahren sind zweifelsohne geeignet, den Staat in seinen Grundstrukturen und seiner Existenz ernsthaft zu bedrohen. [...] Terroristische Aktivitäten mögen zwar unmittelbar nur gewisse Personen an Leib und

22 Statt vieler: BGE 117 Ia 202 E. 5; OFK BV-BIAGGINI, Art. 57 N 5; BSK BV-DIGGELMANN/ALTWICKER, Art. 57 N 4, 24; MOHLER, Föderalismus (Fn.11), S. 7, 11 und 15; SGK BV-SCHWEIZER, Art. 57 N18f.; TIEFENTHAL (Fn.10), §2 N10.

23 Erl. Ber. PMT (Fn. 21), S. 2.

24 Botschaft zum Bundesgesetz über polizeiliche Massnahmen zur Bekämpfung von Terrorismus (Fn.3), S. 4782. Ähnlich im Erl. Ber. PMT (Fn. 21), S. 36. 
Leben gefährden, sollen aber über Kantons- und Landesgrenzen hinausstrahlen und eine Veränderung(en) und Destabilisierung der Gesamtgesellschaft und der grundlegenden Strukturen einer freiheitlichen Demokratie an sich bewirken». «Die Stärkung des präventiv-polizeilichen Instrumentariums im Rahmen des BWIS ist durch die inhärente Kompetenz gedeckt.» ${ }^{25}$

13 Für Bundeszuständigkeiten, für die eine explizite Zuweisung einer Kompetenz an den Bund in der Verfassung fehle, werde nach neuerer Praxis Art.173 Abs. 2 BV herangezogen. ${ }^{26}$ Was das genauer bedeuten soll, bleibt offen. Indessen verweist der Ausdruck auf einen gewohnheitsrechtlichen Bezug («neuere Praxis»).

Diese Praxis steht im Zusammenhang mit der sog. «Dynamisierung» von Art. 57 Abs. 2 BV. ${ }^{27}$ Zum ersten Mal wurde Art. 57 Abs. 2 BV als für den Bund als kompetenzbegründend bei der Revision des Zollgesetzes ${ }^{28}$ vom 18. März 2005 eingeführt. ${ }^{29}$

15 Mit Blick auf das Subsidiaritätsprinzip (Art. $5 a$ und $43 a$ BV) habe der Bund von dieser Kompetenz jedoch schonend Gebrauch zu machen, zumal die Gewährleistung der inneren Sicherheit keine ausschliessliche Bundesaufgabe darstelle. ${ }^{30}$

16 Zudem ortet der Bundesrat ein verfassungsrechtliches Problem, das es zu lösen gelte, im Datenbearbeitungsrecht: «Eine gesamtschweizerische Regelung auf Bundesebene betreffend den interkantonalen und interinstitutionellen Austausch von Personendaten von Gefährderinnen und Gefährdern ist jedoch aus verfassungsrechtlichen Gründen nicht möglich.» Dies zu regeln, sei Kernaufgabe der Kantone. ${ }^{31}$ «Mit Verweis auf den Sicherheitsartikel Art. 57 Abs. 2 BV soll erklärtermassen die innerstaatliche Kompetenzausscheidung im Bereich der Sicherheit beibehalten und keine neue Bundeskompetenz geschaffen werden, die in die Aufgabenbereiche der Kantone eingreift.»32 Daraus soll offenbar abzuleiten sein,

25 Alle dies Zitate: Botschaft zum Bundesgesetz über polizeiliche Massnahmen zur Bekämpfung von Terrorismus (Fn.3), S. 4846.

26 Botschaft zum Bundesgesetz über polizeiliche Massnahmen zur Bekämpfung von Terrorismus (Fn. 3), S. 4845, 4847.

27 Vgl. Bericht des Bundesrates in Erfüllung des Postulats Malama 10.3045 (Fn. 14), S. 4551. Wie die Ausführungen an dieser Stelle im Bericht des Bundesrates in Erfüllung des Postulats Malama 10.3045 zeigen, wurde diese Auslegung des Bundesrates in der Literatur als nicht verfassungskonform einhellig kritisiert. Vgl. MOHLER, Föderalismus (Fn.11), S. 8.

28 Zollgesetz vom 18. März 2005 (ZG; SR 631.0).

29 MOHLER, Föderalismus (Fn.11), S.11.

30 Botschaft zum Bundesgesetz über polizeiliche Massnahmen zur Bekämpfung von Terrorismus (Fn. 3), S. 4846.

31 Erl. Ber. PMT (Fn. 21), S.16. Siehe dazu auch nachfolgend Rz. 61 in fine mit Fn.114.

32 Erl. Ber. PMT (Fn. 21), S. 16. dass der bisher gesetzlich nicht geregelte interkantonale und interinstitutionelle Personendatenaustausch durch die jetzige Regelung gelöst werde.

In der Botschaft wird dann bloss (und widersprüchlich 17 zum Vorherigen) ausgeführt, Art. 23h BWIS ermächtige «fedpol und die zuständigen kantonalen Behörden dazu (...), besonders schützenswerte Personendaten von terroristischen Gefährderinnen und Gefährdern zu bearbeiten». ${ }^{33}$

\section{Zur Erstreckung der strafprozessualen Gesetzgebungskompetenz}

Die zweite Argumentationslinie steht im Zusammenhang 18 mit der Bundeszuständigkeit auf dem Gebiet des Strafund Strafprozessrechts nach Art.123 Abs.1 BV. Gemäss Art. 22 ff. StPO ${ }^{34}$ umfasse die Bundesgerichtbarkeit «u.a. das Gebiet des organisierten Verbrechens und der Terrorismusbekämpfung». ${ }^{35}$ Es folgt: «Die polizeiliche Arbeit im Vorfeld von Strafverfahren, die der Verhinderung, Erkennung und Aufklärung von Straftaten dient, beruht hingegen auf Polizeirecht. Der Bund ist gestützt auf Art. 123 Abs. 1 BV befugt, auch Bestimmungen zur Erfüllung dieser polizeilichen Aufgaben zu erlassen, soweit sie mit der Strafverfolgung von Delikten eng verknüpft sind, die in seiner Strafverfolgungskompetenz liegen. Um solche polizeirechtlichen Normen geht es vorliegend.» ${ }^{36}$

Noch deutlicher wird in der Botschaft ausgeführt: «Von 19 Bedeutung ist sodann die Bestimmung von Art.123 Abs. 1 BV, welche dem Bund auf dem Gebiet des Strafrechts und des Strafprozessrechts Gesetzgebungskompetenzen zuweist. [...] Art.123 Abs. 1 BV ist nicht nur kompetenzbegründend für polizeiliche Aufgaben des Bundes im Rahmen von Strafverfahren, sondern auch für polizeiliches Tätigwerden im Vorfeld von Strafverfahren, das der Verhinderung, Erkennung und Aufklärung von Straftaten dient. Der Bund ist gestützt auf Art.123 Abs.1 BV befugt, auch Bestimmungen zur Erfüllung dieser polizeilichen Aufgaben zu erlassen, soweit sie mit der Strafverfolgung von Delikten eng verknüpft sind, die in seiner Strafverfolgungskompetenz liegen. Die der Strafverfolgung des Bundes unterstellten strafbaren Handlungen umfassen u. a. das Gebiet des organisierten Verbrechens und der Terrorismusbekämpfung.» ${ }^{37}$

\footnotetext{
33 Botschaft zum Bundesgesetz über polizeiliche Massnahmen zur Bekämpfung von Terrorismus (Fn.3), S. 4788.

34 Schweizerische Strafprozessordnung vom 5. Oktober 2007 (Strafprozessordnung, StPO; SR 312.0).

35 Erl. Ber. PMT (Fn. 21), S. 35.

36 Erl. Ber. PMT(Fn. 21), S.35f.

37 Botschaft zum Bundesgesetz über polizeiliche Massnahmen zur Bekämpfung von Terrorismus (Fn. 3), S. 4846.
} 
diese Bestimmungen auch koordinierenden Charakter aufwiesen, könne nebst Art.123 Abs. 1 BV zusätzlich Art. 57 Abs. 2 BV als Verfassungsgrundlage herangezogen werden. ${ }^{38}$

\section{Kritische Anmerkungen dazu} fünf Organisationen das Fehlen einer verfassungsrechtlichen Grundlage fest, sechs Kantone und vier Organisationen beurteilten die Verfassungsrundlage als «bedenklich» (ausg. Ausreisebeschränkungen). ${ }^{39}$ Dies wird in der Zusammenfassung der Vernehmlassungsergebnisse in der Botschaft nicht erwähnt. ${ }^{40}$ Diese Unterlassung verstösst gegen Treu und Glauben (Art. 5 Abs. 3 BV). Wer von den Parlamentsangehörigen nicht auch den gesonderten Bericht über die Vernehmlassungsergebnisse gelesen hat, ist sich der Problematik nach der Lektüre nur der Botschaft, falls in diesen Kompetenzfragen nicht bewandert, nicht bewusst.

Im erläuternden Bericht wird ausgeführt, es gehe darum, eine polizeirechtliche Lücke zu schliessen (vorstehend Rz. 10). Ein gleichlautender Hinweis findet sich auch in der Botschaft. ${ }^{41}$

23 Es wurde (Rz. 8) dargetan, dass die Begründung einer Bundeszuständigkeit durch Lückenfüllung verfassungsrechtlich untersagt ist.

Die inhärente Zuständigkeit des Bundes begründet der Bundesrat damit, dass der Bund «den Bestand des schweizerischen Gemeinwesens zu gewährleisten und für die Abwehr von Gefahren zu sorgen, die dieses existenziell bedrohen» (Rz.12). Damit wird pauschal die Bundeskompetenz in der Terrorismusbekämpfung als Teilbereich des Staatsschutzes vorgegeben. Quasi eingeebnet werden damit zwei unterschiedliche Voraussetzungen für die Begründung und Grenze der Bundeszuständigkeit: Bei der einen handelt es sich um die Art der zu bekämpfenden Gewaltverbrechen, bei der andern um die gesetzlich geregelten Massnahmen dafür.

\footnotetext{
38 Botschaft zum Bundesgesetz über polizeiliche Massnahmen zur Bekämpfung von Terrorismus (Fn. 3), S. 4847.

39 Bericht über das Vernehmlassungsergebnis (19.032), S. 8, 10.

40 Vgl. Botschaft zum Bundesgesetz über polizeiliche Massnahmen zur Bekämpfung von Terrorismus (Fn. 3), S. 4845 f.

41 Botschaft zum Bundesgesetz über polizeiliche Massnahmen zur Bekämpfung von Terrorismus (Fn.3), S. 4769.
}

\section{Zur Typologie der zu bekämpfenden Gewalt- verbrechen}

Der Bundesrat sieht terroristische Aktivitäten grund- 25 sätzlich mit dem Ziel der «Veränderungen und Destabilisierung der Gesamtgesellschaft und der grundlegenden Strukturen einer freiheitlichen Demokratie an sich» verbunden, die sich auf die gesamte Bevölkerung der Schweiz erstreckt und «letztlich die innere Sicherheit der Schweiz als Ganzes» zum Ziel habe. ${ }^{42}$

Dies entspricht jedoch einem traditionellen Terrorismus- 26 begriff, wie er in Art.260quinquies Abs. 3 StGB zum Ausdruck kommt. ${ }^{43}$ Das trifft aber nicht mehr zu. Was unter Terrorismus - nun auch in diesem Gesetz - verstanden wird, weicht vom bisher im Strafrecht Gebräuchlichen ab.

Terrorismus, wie er in den 1970er- und noch 1980er-Jah- 27 ren von der Roten Armee Fraktion (RAF) in Deutschland, den Brigate Rosse (BR) in Italien oder den Cellules Communistes Combattantes (CCC) in Belgien mit schlimmsten Gewaltverbrechen betrieben worden ist, zielte auf einen gewaltsamen Umsturz der verfassungsmässigen Ordnungen. Das traf auch auf al-Qaida mit ihrer Hierarchie samt entsprechenden Befehlen an Unterstellte oder Anhänger zu (z.B. 9/11 in New York 2001, Londoner Anschläge $2005^{44}$ ). Der Islamische Staat (IS) löste sich davon und schuf ein horizontales Netzwerk, ${ }^{45}$ auch wenn sein Gestalter Abu Musab Al-Suri durchaus strategische Gedanken über den Zusammenbruch des Westens durch Bürgerkrieg gehabt haben dürfte. ${ }^{46}$ Die Kämpfer sollten sich allenfalls selber koordinieren, so geschehen 2015 in Paris (Charlie Hebdo und Supermarkt Hyper Cacher). ${ }^{47}$ Doch auch der IS besteht nicht mehr. Heute wirken radikale Hetzer bspw. islamistischer Prägung, die selber keine Gewaltverbrechen begehen. Diese werden individuell von auf diese Weise (z.B. im Gefängnis, im Internet oder in einer Moschee) radikalisierten, nicht notwendigerweise aber organisierten Individuen «bei Gelegenheit» begangen. Das unmittelbare Motiv ist Rache (bspw. wegen einer ihre islamistischen Vorstellungen verletzenden Regelung [z.B. die Laizität, Kopftuchverbot] oder Handlung [missfallende Meinungsäusserung]) oder Hass

42 Botschaft zum Bundesgesetz über polizeiliche Massnahmen zur Bekämpfung von Terrorismus (Fn. 3), S. 4846.

43 «Die Tat gilt nicht als Finanzierung einer terroristischen Straftat, wenn sie auf die Herstellung oder Wiederherstellung demokratischer und rechtsstaatlicher Verhältnisse oder die Ausübung oder Wahrung von Menschenrechten gerichtet ist.» Argumentum e contrario.

44 GILLES KEPEL, Terror in Frankreich - der neue Dschihad in Europa, deutsche Ausgabe München 2016, S. 60.

45 GILLES KEPEL, im Interview der Basler Zeitung vom 4. Januar 2021, S. $4 \mathrm{f}$.

46 KEPEL, Terror in Frankreich (Fn. 44), S. 6of.

47 KEPEL, Terror in Frankreich (Fn. 44), S. $229 \mathrm{ff}$. 
(z.B. auf Andersgläubige). ${ }^{48}$ Es können jedoch auch kaum religiös bedingte Denkmuster Anstoss für Gewaltverbrechen sein (Morges 2020). Ein Umsturz der verfassungsmässigen Ordnung ist jedoch nicht anvisiertes Ziel ihrer Gewaltstraftat.

Das gilt nicht nur für Gewaltverbrechen islamistischer Provenienz, sondern kann auch bspw. auf rechtsextremistische Täter zutreffen. Motiv mag der Hass (weshalb auch immer) auf Minoritäten sein, doch können sie auch zufällige Opfer gewalttätig angreifen (Lugano 2020). ${ }^{49}$ Ein (auch bloss symbolischer) Angriff auf die verfassungsmässige Ordnung ist ausserhalb ihrer mentalen Reichweite. Dennoch nimmt die Bundesanwaltschaft die Ermittlungen auf. 50 ben daher weder eine Intention der Destabilisierung der Gesellschaft noch einer anderen Wirkung, die den Gesamtstaat angehen sollte. Ihre Wirkung in der Bevölkerung ist kaum anders als die Furcht beispielsweise vor einem Raubüberfall auf offener Strasse.

30 Dennoch werden solche Gewaltstraftaten offensichtlich auch als «Terrorverbrechen» eingestuft. Nicht nur landläufig werden (auch) sog. Hassverbrechen ${ }^{51}$ als Terror verstanden, ${ }^{52}$ v.a. gegenüber, freilich nicht nur, Minderheiten. Solche unterscheiden sich aber deutlich von Verbrechen, die auf die Veränderung demokratisch freiheitlicher Strukturen gerichtet sind. ${ }^{53}$

31 Demzufolge beschränkt sich der Teilbereich Terror des Staatsschutzes auf Gewaltstraftaten, die auf die gewaltsame Änderung derverfassungsmässigen Ordnung, aufeine Destabilisierung der gesamten Schweiz oder doch eines erheblichen Teils davon gerichtet sind. Andere Gewaltstraftaten, insbesondere Hassverbrechen oder Delikte psychisch gestörter Personen, fallen nicht darunter. So-

48 KEPEL, Interview (Fn. 45); DERS., Chaos, die Krise in Nordafrika und im Nahen Osten verstehen, deutsche Ausgabe München 2019, S. $420 \mathrm{f}$.

49 So z.B. OLIVER ROY, Woher kommt der jihadistische Terror, NZZ vom 5. Januar 2021, S. 7. Seine von KEPEL teilweise abweichende Aetiologie des sog. dschihadistischen Terrors hat auf die präventive Bekämpfung vordergründig religiös vermuteter Gefährder in diesem Stadium vor der Schwelle mindestens zur (strafbaren) Vorbereitungshandlung oder dann zum Versuch keinen Einfluss. Sie kann in einem früheren Stadium je nach Umständen bedeutungsvoll sein.

50 Medienmitteilung der Bundesanwaltschaft vom 14. September 2020; Tagesanzeiger vom 25. November 2020

51 Als Beispiele etwa die Attentate von Christchurch/NZ (15. März 2019) oder Halle (9. Oktober 2019).

52 Ein Gesetzestext darf sich nicht zu weit von der Parallelwertung in der Laiensphäre entfernen (BGE129 IV 238 E.3.2.2). Er soll diese berücksichtigen, ohne dadurch an Bestimmtheit einzubüssen. Dies gilt auch umgekehrt.

53 Vgl. dazu Bericht des Bundesrates in Erfüllung des Postulats Malama 10.3045 (Fn. 14), S. 4487. Siehe dazu eingehend MÜLLER (Fn. 21), S. $404 \mathrm{f}$. mit entfällt dafür auch die Zuständigkeit des Bundes in solchen Fällen.

Umgekehrt heisst dies, dass die Kantone bei den Ermitt- 32 lungen, sei es präventiver oder repressiver Art, auf mögliche Verbindungen, die auf weitergehende Pläne oder Verbindungen hindeuten könnten, achten müssen.

\section{Zur Typologie der Massnahme}

Selbst wenn für einen Anschlag das Motiv eines Angriffs 33 auf die verfassungsmässige Ordnung oder die Destabilisierung der Gesellschaft angenommen wird, beschränkt sich die Zuständigkeit des Bundes bei deren präventiven Bekämpfung auf die Informationsbeschaffung (Rz.6). Es geht um informationelle Realakte, von welchen die Zielperson physisch in ihrer (Bewegungs-/Handlungs-)Freiheit nicht eingeschränkt wird. Sicherheitspolizeiliche operationell ${ }^{54}$ intervenierende Realakte $^{55}$ und deren gesetzliche Regelung fallen nicht in die Bundeskompetenz. Das gilt im präventiven Bereich auch für die entsprechenden Verfügungen. Die Mittel der Verhinderung von Straftaten beschlägt das Polizeirecht, wofür die Kantone zuständig sind, was der Bundesrat andernorts selber festgehalten hat. ${ }^{56}$ Es geht auch aus Art. 4 Abs. 1 BWIS hervor. Dies entspricht ebenso der Praxis des Bundesgerichts. ${ }^{57}$

Demnach gebrichtes dem Bund auch an der Kompetenz im 34 Teilbereich Terrorismusbekämpfung des Staatsschutzes zur Gesetzgebung sicherheitspolizeilicher operationell intervenierender Realakte und deren Anordnung. Das ergibt sich auch aus Art. 6 Abs. 2 (informieren und alarmieren der zuständigen staatlichen Stellen) und Art. 8 Abs. 2 NDG (Bewaffnung ausschliesslich für Notwehr und Notstand). Die angestrebte «Stärkung des präventiv-polizeilichen Instrumentariums im Rahmen des BWIS» (Rz.6) kann ausschliesslich die Informationsbeschaffung betreffen.

54 Im Gegensatz zu informationell intervenierend wie bspw. die Überwachung des Post- und Fernmeldeverkehrs, der Einsatz von besonderen technischen Geräten zur Überwachung des Fernmeldeverkehrs und von Ortungsgeräten oder von Überwachungsgeräten, um das nicht öffentlich gesprochene Wort abzuhören (Art. 26 Abs. 1 lit. a bis c NDG).

55 MARKUS H.F. MOHLER, Grundzüge des Polizeirechts, Basel 2012 N 861 ff.; DERS., Polizeirecht (Fn. 8), S. 67 f.

56 Stellungnahme des Bundesrats vom 23. Mai 2012 zum Bericht der Kommission für Rechtsfragen des Nationalrates vom 3. Februar 2012 zur Parlamentarischen Initiative «Präzisierung des Anwendungsbereichs der Bestimmungen über die verdeckte Ermittlung» (BBl 20125609), S. 5611. A.A. wohl TIEFENTHAL (Fn. 10), \$2 N33f., der eine umfassende Bundeskompetenz im Staatsschutz sieht, ohne aber nach den - auch gemäss den Gesetzestexten - unterschiedlichen Massnahmen bzw. Mitteln zu unterscheiden oder auf Art. 4 Abs.1BWIS einzugehen.

57 BGE140 I353 E. 5.1: «Soweit dagegen zu regeln ist, mit welchen Mitteln Straftaten verhindert werden können oder ihre erst mögliche Begehung festgestellt werden kann, beschlägt dies das Polizeirecht, zu dessen Erlass grundsätzlich die Kantone zuständig sind.» 
a) Zur angeführten Verfassungsgrundlage von Art. 173 Abs. 2 BV Praxis» auch Art. 173 Abs. 2 BV zur Begründung einer verfassungsrechtlichen Grundlage für eine polizeirechtliche Gesetzgebung an (Rz.13). Im Bericht Malama wird diese Verfassungsbestimmung einerseits nur als Organkompetenz verstanden, ${ }^{59}$ andererseits entweder historisch oder bloss aufzählend, ohne materielle Begründung erwähnt. Beim Bundesgesetz über die privaten Sicherheitsdienstleistungen im Ausland gehört die Nennung von Art. 173 Abs. 2 BV ohnehin zu den «unorthodoxen Abstützversuchen».60

Art. 173 Abs. 2 BV scheidet als Verfassungsrundlage für eine (u.a.) polizeirechtliche Gesetzgebung aus zwei Gründen aus: Zum einen - mit Bezug auf die «neuere Praxis» - kann Gewohnheitsrecht keine Bundeskompetenz begründen (s. Rz. 8), zum anderen legt diese Bestimmung lediglich die Organkompetenz der Bundesversammlung innerhalb der Bundesorgane - im Verhältnis zu Art. 184 und 185 BV (Kompetenzen des Bundesrates) - fest, nicht aber Bundeskompetenzen im Verhältnis zu den Kantonen. ${ }^{61}$

\section{b) Zur angeführten Verfassungsgrundlage von Art. 57 Abs. 2 BV}

37 Soweit die Bestimmungen koordinierenden Charakter hätten, könne «zusätzlich Art. 57 Abs. 2 BV als Verfassungsgrundlage herangezogen werden».62 Dass Art. 57 Abs. 2 BV keine Bundeskompetenz begründet, sondern ausdrücklich «Bund und Kantone im Rahmen ihrer $\mathrm{Zu}$ ständigkeiten für die Sicherheit» sorgen lässt, ist in Lehre und Rechtsprechung unbestritten. ${ }^{63}$ Das Gegenteil stünde auch im direkten Widerspruch zur Botschaft über die

58 Vgl. Ingress zum Bundesgesetz über die polizeilichen Informations systeme des Bundes vom 13.Juni 2008(BPI; SR 361), vgl. dazu Bericht des Bundesrates in Erfüllung des Postulats Malama 10.3045 [Fn.14], S. 4495, zum vom NDG abgelösten Bundesgesetz vom 3. Oktober 2008 über die Zuständigkeiten im Bereich des zivilen Nachrichtendienstes (ZNDG, ehem. SR121); (Bericht des Bundesrates in Erfüllung des Postulats Malama 10.3045, S. 4496), zum Bundesgesetz über die im Ausland erbrachten privaten Sicherheitsdienstleistungen vom 27. September 2013 (BPS; SR 935.41); (Bericht des Bundesrates in Erfüllung des Postulats Malama 10.3045 [Fn.14], S. 4498) und zum NDG.

59 Bericht des Bundesrates in Erfüllung des Postulats Malama 10.3045 (Fn.14), S. 4476.

60 OFK BV-BIAGGINI, Vorbem. Art. 42-135N19, mit weiteren Beispielen.

61 Das ergibt sich schon aus der Systematik der BV. Dazu: OFK BV BIAGGINI, Vorbem. Art. 42-135 N19; DANIEL MOECKLI, in: Diggelmann/Randall/Schindler (Hrsg.), Verfassungsrecht der Schweiz, Bd.3, Zürich 2020, VIII/8 N 10, S. 2263; PIERRE TSCHANNEN, Normarchitektur des Bundes, in: Kiener/Bühler/Schindler (Hrsg.), Sicherheits- und Ordnungsrecht des Bundes, Bd. III, Teil 2, Basel 2018, N13.

62 Botschaft zum Bundesgesetz über polizeiliche Massnahmen zur Bekämpfung von Terrorismus (Fn. 3), S. 4847, vgl. vorstehend Rz. 20.

63 Statt vieler: BSK BV-DIGGELMANN/ALTWICKER, Art. 57 N 24 in fine; MOECKLI, Verfassungsrecht (Fn. 61), N 9; SGK BV-SCHWEIZER/MOHLER, Art. 57 N3.
Bundesverfassung. ${ }^{64}$ Diese Zuständigkeit wird vom Bund in Gesetzesvorhaben ausschliesslich selbstreferenziell begründet.

\section{c) Subsidiaritätsprinzip}

Widersprüchlich ist die Botschaft des Bundesrates hin- 38 sichtlich des Subsidiaritätsprinzips (Art. $5 a$ und $43 a$ BV, siehe Rz. 7, 15). Dem zweifach sich selber anmahnenden «schonenden Gebrauch» der Bundeskompetenzen ${ }^{65}$ widerspricht der gesetzliche Erlass sicherheitspolizeilicher Massnahmen explizit. Weniger «schonend» ist nicht mehr möglich, handelt es sich doch um einen eigentlichen Einbruch in diegesetzgeberische Zuständigkeit der Kantone. ${ }^{66}$ Dass die Kantone nach Art. 23r BWIS mit dem Vollzug beauftragt werden, entspricht lediglich der verfassungsrechtlichen Regelung nach Art. 46 Abs. 1 BV. ${ }^{67}$ Adressat des neu in der BV explizit stipulierten Subsidiaritätsprinzips ist der Bundesgesetzgeber; er hat die Aufgabe, dieses «von Fall zu Fall zu konkretisieren». ${ }^{68}$ Dem Subsidiaritätsprinzip direkt entgegen haben Bundesrat und Parlament dieses Gesetz in der vorliegenden Form erlassen.

\section{d) Gesetzgeberischer Widerspruch innerhalb des BWIS}

Nach Art. 4 Abs. 1 BWIS sind die Kantone für die innere 39 Sicherheit ihres Gebietes ist in erster Linie selber verantwortlich. Demzufolge sind sie auch für die dafür notwendige Gesetzgebung zuständig. Die mit dem PMT-Gesetz ins BWIS eingefügten Normen zur Festlegung eines abschliessenden Massnahmenkataloges unterlaufen die kantonale Gesetzgebungskompetenz damit direkt und verletzen daher auch das Subsidiaritätsprinzip (vorstehend).

\section{e) Zur Datenbearbeitungsfrage}

Ferner spricht der Bundesrat eine (vermeintliche) Daten40 bearbeitungsproblematik an (Rz.16).

Die Datenbearbeitung durch kantonale (und kommunale) 41 Polizeidienste innerhalb des Kantons ebenso wie der Datenaustausch zwischen den Kantonen und dem Bund durch die kantonalen Dienste sind im kantonalen Recht, vorab in den Polizeigesetzen, geregelt. Dafür bedarf es -

64 Botschaft vom 20. November 1996 über eine neue Bundesverfassung (BBl1997 I1), S. 237.

65 Botschaft zum Bundesgesetz über polizeiliche Massnahmen zur Bekämpfung von Terrorismus (Fn. 3), S. 4846, 4848.

66 MOECKLI (Fn.61), N19 (S. 2266); MOHLER, Grundzüge (Fn. 55), N194 ff.

67 ZIMMERLIN (Fn. 6), S. 189, sieht den Grund dafür darin, dass der Bund keine genügende Verfassungsgrundlage habe. Das trifft m.E. insofern nicht zu, da der Vollzug gemäss Art. 46 Abs. 1 BV nach Massgabe von Verfassung und Gesetz den Kantonen obliegt und der Bund für den Vollzug der Massnahmen ohnehin die Mittel nicht hat.

68 Botschaft zur Neugestaltung des Finanzausgleichs und der Aufgaben zwischen Bund und Kantonen (NFA) vom 14. November 2001 (BBl 20022291), S. 2339. Vgl. auch TSCHANNEN, Normarchitektur (Fn.14), N14. 
neben den Bestimmungen des BPI und des NDG - keiner weiteren gesetzlichen Grundlagen. Diese führten in der ohnehin schon unübersichtlichen datenrechtlichen Regelung in der Schweiz ${ }^{69} \mathrm{zu}$ weiteren Unklarheiten in Form möglicherweise konfligierender Bestimmungen mit Regelungen kantonaler Bedrohungsmanagements.

(siehe Rz.16) lassen die Vermutung aufkommen, der Bundesrat spreche Art. $43 a$ Abs. 1 BV an, wonach der Bund - als ein Grundsatz der Subsidiarität - Aufgaben übernehme, welche die Kraft der Kantone übersteigen oder einer einheitlichen Regelung bedürfen. Dies trifft jedoch nicht zu, da dieZuständigkeiten für die Datenbearbeitung im bundesstaatlichen System durch Art. 2 Abs. 1 DSG $^{70}$ und in den kantonalen Datenbearbeitungs- und Polizeigesetzen ebenso wie durch das BPI und das NDG mit seinen Verordnungen geregelt sind.

\section{f) Zur Verfassungsgrundlage von Art. 123 Abs. 1} BV für sicherheitspolizeiliche Massnahmen

43 Diese im erläuternden Bericht ${ }^{71}$ und in der Botschaft ${ }^{72}$ dargelegte Ausweitung der Bundeszuständigkeit ins Vorfeld von Straftaten, d.h. bevor ein hinreichender Tatverdacht für die Eröffnung eines Ermittlungsverfahrens angenommen wird, überrascht und widerspricht der bisherigen Kompetenzaufteilung. Auf diese Zuständigkeitsbegründung ist der Bundesrat auch im erwähnten Bericht zum Postulat Malama nicht gekommen. Sie entspricht der von RHINOW ET AL. beklagten schleichenden Ausweitung von Bundeskompetenzen. ${ }^{73}$

44 Nach bisher unbestrittener Zuständigkeitsgliederung gehört die Regelung der Mittel zur Verhinderung von Straftaten zum Polizeirecht und ist damit Sache der Kantone (Rz.9).

Träfe die jetzige Argumentation des Bundesrates zu, erwiesen sich alle in jüngerer Zeit just wegen der nicht gegebenen Gesetzgebungskompetenz des Bundes in kantonale Polizeigesetze eingefügten Bestimmungen über die verdeckten Vor-Ermittlungen und die verdeckte Fahndung ${ }^{74},{ }^{75}$ wegen der nachträglich derogatorischen Wirkung von Art. 123 Abs. 1 BV76 als bundesrechtswidrig. Die

69 Vgl. EDÖB, Jahresbericht 2018/19, S. 29; MOHLER, Polizeirecht (Fn. 8), S.129ff.

70 Bundesgesetz über den Datenschutz vom 19.Juni 1992 (DSG;SR 235.1)

71 Erl. Ber. PMT (Fn. 21), S. 35f.

72 Botschaft zum Bundesgesetz über polizeiliche Massnahmen zur Bekämpfung von Terrorismus (Fn. 3), S. 4846.

73 RHINOW/SCHEFER/UEBERSAX (Fn. 10), N696.

74 Z.B. Art.111 ff. und 114 ff. PolG/BE (BSG 551.1); \$\$ 33 a und 33b PolG/BS (SG 550.1); Art. 56 ff. PolG/GE (RS/GE F 1.05); \$32e PolG/ZH (OS 551.1).

75 Präventive Massnahmen, die gerade nicht von Art. $285 a$ ff. und 298 aff. StPO gedeckt sind.

76 Vgl. SGK BV-vEST, Art. 123 N 2 und 8.
Argumentation des Bundesrates geht nach der hier vertretenen Auffassung gänzlich fehl. Das Bundesgericht hat solche Bestimmungen in kantonalen Polizeigesetzen bereits wiederholt als bundesrechtskonform beurteilt ${ }^{77}$ und damit deutlich zwischen der straf-/strafprozessund der sicherheitspolizeirechtlichen Gesetzgebungskompetenz unterschieden.

\section{g) Kritik an der fehlenden Verfassungskompe- tenz im Vernehmlassungsverfahren}

Die fehlende Bundeszuständigkeit zum Erlass dieses Ge- 46 setzes wurde in Vernehmlassungen ${ }^{78}$ unter mehreren Gesichtspunkten angesprochen. Auch wurde festgehalten, dass die derzeit bestehenden Möglichkeiten zur Verhinderung eines terroristischen Angriffs noch nicht genügten. Diese Lücke mit den neuen BWIS-Bestimmungen im PMT-Gesetz zu füllen, geht jedoch verfassungsrechtlich nicht an.

Der Novellierung des BWIS in diesem PMT-Mantelgesetz 47 gebricht es an der notwendigen Verfassungsgrundlage, dem Bund fehlt die Zuständigkeit für deren Erlass. ${ }^{79}$

\section{Abschliessende Betrachtungen}

Bemühungen, für die präventive Bekämpfung von Ge- 48 waltstraftaten mit einem Bezug zu Terrorismus oder gewalttätigem Extremismus, einschliesslich entsprechend motivierte Hassverbrechen, wirksame gesetzliche Grundlagen in der ganzen Schweiz zu schaffen, sind notwendig. Die bundesstaatliche Kompetenzgliederung bereitet diesen Bestrebungen bisher jedoch verfassungsrechtliche Schwierigkeiten.

\section{Folgen für Haftung und kantonale Gesetzgebung}

Zunächst zeitigt dieses PMT-Gesetz, soweit das BWIS da- 49 durch geändert wird, zwei wohl nicht beachtete Folgen:

Wie erwähnt sind die Kantone nach Art. $23 r$ Abs.1 BWIS 50 für den Vollzug der Massnahmen zuständig und damit verantwortlich. Wie im zweiten Teil dieser Ausführungen noch zu zeigen sein wird, erheischen die Massnahmen, sofern sie die beabsichtigte Wirkung erreichen sollen, einen erheblichen Aufwand an Ressourcen (Personal und Mittel). Selbst grosse Polizeidienste dürften für die vorgesehene Dauer solcher Massnahmen (zwölf bzw. neun Monate) nicht über die nötigen Kapazitäten

77 Urteil des Bundesgerichts 1C 181/2019 vom 29. April 2020 E.15.2; BGE140I353 E. 5.1, 8.2.

78 So z.B. in derjenigen der International Commission of Jurists, Sek tion Schweiz.

79 Vgl. auch EDÖB, Jahresbericht 2019/20, S. 28. 
verfügen. Kommt es daher trotz der Anordnung solcher Massnahmen zu einer Gewaltstraftat mit Opfern, haftet der mit dem Vollzug derselben beauftragte Kanton. Der Bund haftet nach dem Verantwortlichkeitsgesetz ${ }^{80}$ nicht. Art. 1 Abs. 1 lit. f VG ${ }^{81}$ erstreckt sich nicht auf kantonale Amtsträger, auch wenn diese mit öffentlich-rechtlichen Aufgaben des Bundes betraut sind. ${ }^{22}$ Damit kann ein Kanton zufolge ungenügender Präventionsmassnahmen mit Schadenersatz- und Genugtuungsansprüchen konfrontiert werden, zu denen er die Kräfte und Mittel nicht hatte und deren Wirkungslosigkeit ohne diese Ressourcen von vorneherein feststand. ${ }^{83}$ Solche Forderungen könnten sehr hoch ausfallen.

Die zweite Folge betrifft die Beeinträchtigung der kantonalen Gesetzgebungskompetenz im Polizeirecht ${ }^{84} \mathrm{bzw}$. eine «Souveränitätsbeschränkung» der Kantone durch ein kompetenzwidrig erlassenes Bundesgesetz. ${ }^{85} \mathrm{Nach}$ Art. 49 Abs. 1 BV geht Bundesrecht entgegenstehendem kantonalen Recht vor. Diese derogatorische Kraft des Bundesrechts ${ }^{86}$ bewirkt, dass bei gleichem Regelungsgegenstand, hier: freiheitsbeschränkende polizeirechtliche Massnahmen zur präventiven Bekämpfung bestimmter Gewaltstraftaten, Bundesrecht kantonalem Recht vorgeht. ${ }^{87}$ Der Massnahmenkatalog im novellierten BWIS ist abschliessend. ${ }^{88}$ Weitere Freiheitsbeschränkungen mit dem gleichen Ziel können Kantone nicht vorsehen, ohne das bundesgesetzliche Konzept zu beeinträchtigen. Vorbehalten bleiben nach Art. 23r BWIS nur kantonalrechtlich geregelte polizeiliche Zwangsmassnahmen zu deren Durchsetzung. Das bedeutet in der Praxis, dass vorbestehende kantonale polizeigesetzliche Regelungen mit dem gleichen Zweck ungültig89 werden, umgekehrt, dass die Kantone nicht mehr befugt sind, entsprechende Bestimmungen zu erlassen. ${ }^{90}$

80 Bundesgesetz über die Verantwortlichkeit des Bundes sowie seiner Behördenmitglieder und Beamten vom 14. März1958(VG; SR 170.32).

81 «Den Bestimmungen dieses Gesetzes unterstehen alle Personen, [...] nämlich: alle anderen Personen, insoweit sie unmittelbar mit öffentlich-rechtlichen Aufgaben des Bundes betraut sind.»

82 Botschaft zum Entwurf eines Verantwortlichkeitsgesetzes, vom 29. Juni 1956 (BBl1956 I1393), S. 1398f.; Urteil des Bundesgerichts 2C_806/2020 vom 29. September 2020 E. 2.2.

83 Siehe dazu die Ausführungen in der folgenden Darlegung.

84 SGK BV-RUCH, Art. 49 N19.

85 OFK BV-BIAGgINI, Art. 3 N 12.

86 OFK BV-BIAGGINI, Art. $49 \mathrm{~N} 2$.

87 Vgl. dazu BSK BV-WALDMANN, ART. 49 N 5. Auf die dogmatische Unterscheidung zwischen «Vorrang» und derogatorische Kraft des Bundesrechts wird hier nicht weiter eingegangen.

88 Vgl. betreffend «abschliessend» BGE131I223 E. 3.3 ff.

89 Auf die dogmatische Diskussion, ob sie überhaupt keine Rechtswirkung entfalten, d.h nichtig oder bloss anfechtbar sind, wird hier nicht eingetreten.

90 BGE143I403 E. 7.1; Urteil des Bundesgerichts 4C_1/2013 vom 25.Juni 2013 E. 4.1.2; SGK BV-RUCH, Art. 49 N12.

\section{Zum Fehlen einer effektiven Verfassungsge- richtsbarkeit gegenüber Bundesgesetzen}

Zwar gilt diese Vorrangregelung für Bundesrecht nur, 52 sofern dieses gestützt auf eine verfassungsmässige Kompetenz erlassen worden ist. ${ }^{91}$ Diese auf die verfassungsmässige Zuständigkeit gestützte Beschränkung des Vorranges von Bundesrecht wird in Bezug auf Bundesgesetze durch Art. 190 BV aufgehoben: Für das Bundesgericht sind u.a. Bundesgesetze massgebend, eine Prüfung auf deren Verfassungsmässigkeit mit rechtlichen Folgen bei einer Verneinung steht ihm nicht zu. ${ }^{92}$ Die kompetenzwidrig erlassenen PMT-Bestimmungen im BWIS ändern daher wegen der Massgeblichkeit von Bundesgesetzen nach Art.190 BV nichts daran, dass die Kantone in ihrer diesbezüglichen Rechtsetzungszuständigkeit verfassungswidrig eingeschränkt sind.

Art. 190 BV enthält in Bezug auf Bundesgesetze ein An- 53 wendungsgebot, aber kein Prüfverbot.93 Gestützt auf Art. 189 Abs. 3 BV und Art.120 Abs. 1 lit. a des Bundesgerichtsgesetzes ${ }^{94}$ können Kantone auf Feststellung und somit auf einen Kompetenzkonflikt klagen. ${ }^{95}$ Eine direkte Rechtswirkung hätte ein Urteil auch im Falle der Bejahung der fehlenden Verfassungsgrundlage für die Änderungen im BWIS jedoch nicht.

\section{Das PMT-Gesetz ist kein Einzelfall: Die wiederholte Missachtung der bundes- staatlichen Zuständigkeitsordnung im Sicherheits- und Polizeirecht}

Dieses Gesetz ist indessen nur der jüngste Beleg, dass 54 das föderalistische System im Sicherheits- und Polizeirecht in der bestehenden Form an seine Grenzen gestossen ist und verfassungskonforme Weiterentwicklungen für gesamtschweizerische Regelungen nur unbefriedigend (z.B. durch gesamtschweizerische Konkordate) oder gar nicht zulässt.

Die hier diskutierte Gesetzgebung zeigt deutlich, dass 55 die derzeitige Kompetenzaufteilung zwischen Bund und

91 HÄFELIN ET AL. (Fn.10), S.1180; RHINOW/SCHEFER/UEBERSAX (Fn.10), S. 745f., 751; SGK BV-RUCH, Art. 49 N 5; TSCHANNEN, Staatsrecht (Fn.10), §22 N18.

92 OFK BV-BIAGGINI, N12 zu Art. 3; HÄFELIN et al. (Fn.10), N1180; TSCHANNEN, Staatsrecht (Fn. 10), \$22 N18; BSK BV-WALDMANN, Art. $49 \mathrm{~N} 9$.

93 MOHLER, Föderalismus (Fn. 11), S. 45

94 Bundesgesetz über das Bundesgericht vom 17.Juni 20015 (BGG; SR173.110).

95 OFK BV-BIAGGINI, Art. 189 N10; HÄFELIN et al. (Fn.10), N1202; RHINOW/SCHEFER/UEBERSAX (Fn.10), N760f.; FELIX UHLMANN, Gerichtliche Überprüfung der föderalistischen Grundsätze in der Schweiz, Gutachten vom 18. Juli2016 z.H.d. Konferenz der Kantonsregierungen (KDK), N 8; TSCHANNEN, Staatsrecht (Fn.10), §22 N60. 
Kantonen im Sicherheits- und Polizeirecht weder den zu stellenden Anforderungen von der Bewältigung neuerer Formen der Schwerkriminalität (bei Weitem nicht nur in Bezug auf Gewaltdelikte) noch von der zersplitterten Rechtslage, der Koordination und einem verfassungsgemässen ${ }^{96}$ kostengünstigen Ressourceneinsatz her zu genügen vermag. ${ }^{97}$ Dies führte zu einer unerfreulichen rechtlichen Gemengelage in Bezug auf exekutiv-polizeiliche Kompetenzen und deren Grenzen von Bundesorganen wie auch in Bezug auf die Rechtswege beispielsweise bei gemischt auftretenden Einsatzkräften. ${ }^{98}$ So wurde den kantonalen Polizeidiensten bspw. beim Vollzug des Ausländerrechts die Anwendung der Bestimmungen des Zwangsmassnahmengesetzes ${ }^{99}$ statt des eigenen Polizeigesetzes vorgeschrieben, wofür es weder sachlich noch rechtlich eine hinreichende Begründung gibt. Auch dem Bundesgesetz über die Sicherheitsorgane der Transportunternehmen im öffentlichen Verkehr ${ }^{100}$ fehlt eine verfassungsrechtliche Grundlage; die Aufgabe könnte durch Stadtund Kantonspolizeidienste erfüllt werden, was nun jedoch von einer rechtlich gesehen privaten «Polizei» («SBBPolizei») als Organ der SBB AG getan wird. ${ }^{101}$ Gemäss diesem Erlass unterstehen städtische oder kantonale Polizeidienste, die in städtischen oder regionalen Tram-oder Busbetrieben die gesetzlich geforderte Aufgabe erfüllen, der Aufsicht des Bundesamtes für Verkehr und nicht ihren vorgesetzten Behörden. ${ }^{102}$ Das Militärgesetz ${ }^{103}$ wurde hinsichtlich der Voraussetzungen für Assistenzdiensteinsätze zugunsten ziviler Behörden in einer Art. 58 Abs. 2 BV explizit widersprechenden Weise geändert.104

56 Das neuste Beispiel ist der Vorentwurf zu einem Bundesgesetz über den Allgemeinen Teil der Abgabenerhebung

96 Unzulässig ist in diesem Zusammenhang auch die Rückübertragung kantonaler Aufgaben an den Bund, wie das Art.97 ZG vorsieht und nun auch wieder im VE zum BAZG-VG (Fn.106) vorgesehen ist. Die Kompetenzverteilung ist verfassungsrechtlich zwingend; sie kann weder durch einen einseitigen Akt noch durch Vertrag geändert werden. So schon PETER SALADIN, in: Aubert/ Eichenberger/Müller/Rhinow/Schindler (Hrsg.), Kommentar zur aBV, Art. 3 N 164; HÄFELIN et al. (Fn. 10), N1062; RHINOW/SCHEFER/ UEBERSAX (Fn.10), N901f.;TSCHANNEN, Staatsrecht (Fn.10), §19 N 5

97 Zum Ganzen: MOHLER, Föderalismus (Fn.11), S. 45 ff.

98 Kantonale Polizei und GWK- oder Armeeangehörige.

99 Bundesgesetz über die Anwendung polizeilichen Zwangs und polizeilicher Massnahmen im Zuständigkeitsbereich des Bundes vom 20. März 2008 (ZAG; SR 364).

100 Bundesgesetz über die Sicherheitsorgane der Transportunternehmen im öffentlichen Verkehr vom 18. Juni 2010 (BGST; SR 745.2).

101 Vgl. MARKUS MOHLER, Kurzkommentar zum Bundesgesetz über die Sicherheitsorgane der Transportunternehmen im öffentlichen Verkehr (BGST) vom 18. Juni 2010, Jusletter vom 6. September 2010; MOHLER, Föderalismus (Fn.11), S.33 (nach Auskunft von BAV und SBB ist diese Polizei eine Organisation ohne eine definierte Rechtsform).

102 Art.8BGST.

103 Bundesgesetz über die Armee und die Militärverwaltung vom 3. Februar 1995 (MG; SR 510.10).

104 Art. 67 Abs. 1 lit. a MG. und die Kontrolle des grenzüberschreitenden Warenund Personenverkehrs ${ }^{105}$ durch ein neu zu schaffendes Bundesamt für Zoll und Grenzsicherheit. ${ }^{106}$ Dieses Konzept sieht eine in der ganzen Schweiz zuständige Sicherheits- und Kriminalpolizei vor, die sich neben ihren nach Art. 133 BV zoll- und abgaberechtlich zukommenden Aufgaben auch mit der «grenzüberschreitende(n) Kriminalität und illegaler Migration», der «Wahrung der inneren Sicherheit und zum Schutz der Bevölkerung» befasst. ${ }^{107}$ Als BV-Grundlage werden Art. 57 und Art. 133 BV angeführt. ${ }^{108}$ Dass für die nicht zoll-, abgabe- und grenzpolizeilich angestrebten Befugnisse keine verfassungsmässige Zuständigkeit besteht, wird ignoriert. ${ }^{109}$

Umgekehrt sind nur wenige Kantone in der Lage, schwe- 57 re Verbrechensformen (Formen der organisierten Kriminalität, Cybercrime) präventiv und strafrechtlich erfolgreich zu bekämpfen, insbesondere was die Holkriminalität angeht. Das gilt teilweise auch für die Bekämpfung von Gewaltstraftaten mit Bezug zu Terrorismus oder Gewaltextremismus.

Die Politik sowohl von Bund und Kantonen haben die 58 mannigfachen Problemimpulse nicht in ihrer Tiefe wahrgenommen oder verdrängt. Stattdessen werden bei dringenden Problemen neben den bestehenden Konferenzen auf politischer und Chefbeamten-Ebene neue «Plattformen» geschaffen, ${ }^{110}$ die keine gesetzlichen Grundlagen haben und alle keine bindenden Beschlüsse fassen können.

Zudem wird versucht, nötige gesamtschweizerische $m a-59$ teriell-rechtlich gesetzgebende Regelungen durch Konkordate zu erzielen, ${ }^{111}$ was bisher nicht gelungen ist. ${ }^{112}$

105 Ein bemerkenswert irreführender Titel im Vergleich zum über das Zoll- und Abgaberecht hinausgehenden ebenso beabsichtigten Inhalt des Gesetzes.

106 Bundesgesetz über den Allgemeinen Teil der Abgabenerhebung und die Kontrolle des grenzüberschreitenden Waren- und Personenverkehrs durch das Bundesamt für Zoll und Grenzsicherheit (BAZG-Vollzugsaufgabengesetz, BAZG-VG); Abgeschlossene Vernehmlassungen des eidgenössischen Finanzdepartements.

107 Art.1 lit.b VE BAZG-VG.

108 Erläuternder Bericht zum BAZG.VG vom 11. September 2020, S. 203.

109 S. RAINER J. SCHWEIZER / MARKUS H.F. MOHLER, Die polizeilichen Ausgleichsmassnahmen des Bundes und der Kantone nach dem Wegfall der Personenkontrolle an der Landesgrenze in verfassungsrechtlicher Sicht, in: Breitenmoser/Gless/Lagodny (Hrsg.), Schengen in der Praxis, Erfahrungen und Ausblicke, Zürich et al. 2009, S. $120 \mathrm{ff}$.

110 Vgl. die nicht vollständige Auflistung in MOHLER, Föderalismus (Fn.11), S.40 ff.

111 Vgl. zum Ganzen: FELIX UHLMANN / viTAL ZEHNDER, Rechtsetzung durch Konkordate, LeGes 1/2011, S. $9 \mathrm{ff}$.

112 So sind dem Konkordat über Massnahmen gegen Gewalt anlässlich von Sportveranstaltungen vom 15. November 2007 alle Kantone beigetreten, nicht jedoch die beiden Basel der Fassung vom 2. Februar 2012 (Stand: 5. Februar 2021). Auch der Interkantonalen Vereinbarung (bzw. Konkordat) über die computergestützte 


\section{Folgen und Reformbedarf}

Die seit einigen Jahren im Sicherheits- und Polizeirecht zu beobachtende Entwicklung der bundesrechtlichen Gesetzgebung kommt einer Aushöhlung der bundesstaatlich Zuständigkeitsordnung gleich. Dabei geht es nicht nur um Bundesgesetze, denen die Verfassungsgrundlage fehlt, sondern auch um einzelne Regelungen, die unmittelbar in die kantonale Polizeihoheit eingreifen. Neben der bereits erwähnten aufsichtsrechtlichen Unterstellung kantonaler und städtischer Polizeidienste unter das BAV, sofern sie polizeiliche Aufgaben im öffentlichen Verkehr wahrnehmen (Rz. 55), werden im Zwangsmassnahmengesetz auch die Aus- und Weiterbildungsprogramme für die in seinem Geltungsbereich eingesetzten Polizeikräfte ebenso wie die Beschaffung von Zwangsmitteln verbindlich geregelt.113 Umgekehrt fehlen Rechtsschutzbestimmungen, was in gleichen oder doch gleichgelagerten Fällen zu unterschiedlichen Verfahrenswegen und Zuständigkeiten führt.

Zusammenarbeit der Kantone bei der Aufklärung von Gewaltdelikten vom 02. April 2009 (ViCLAS-Konkordat) sind nicht alle Kantone beigetreten. Ein Konkordat über private Sicherheitsdienstleistungen vom 12. November 2010 ist nicht zustande gekommen (siehe auch Homepage der Konferenz der Kantonalen Justiz- und Polizeidirektorinnen und-direktoren). Eine Interkantonale bzw. interbehördliche Vereinbarung der Nordwestschweizer Kantone über den Datenaustausch zum Betrieb von Lage- und Analysesystemen im Bereich der seriellen Kriminalität vom 14.Juni ist als rechtsetzendes Konkordat angenommen worden (siehe bspw. SGS/BL 700.16). Gemäss Art. 17 Abs. 1 dieses Konkordates können ihm alle Kantone und «geeignete Bundesstellen» beitreten .

113 Näheres dazu in MOHLER, Föderalismus (Fn.11), S. 32.
Im Ergebnis hat diese Rechtsentwicklung zu einer ver- 61 fassungswidrigen und überkomplexen Rechtslage geführt mit kaum auflösbaren Vorschriftengemengen. Bereitet dies schon rechtsanwendenden Behörden erhebliche Schwierigkeiten, ${ }^{114}$ so ist es für die Bevölkerung unmöglich geworden festzustellen, welche polizeilich tätigen Dienste weshalb, wo, gestützt worauf zu welchen grundrechtlichen Beschränkungen befugt sind. Dies widerspricht dem Legalitätsprinzip in Bezug auf die Kriterien der Klarheit, Verständlichkeit, Transparenz und Zugänglichkeit massgebender Normen.

Eine Teilrevision der Sicherheitsverfassung ist dringend. 62 Dabei geht es keineswegs um eine Eliminierung des Föderalismus im Polizeirecht, im Gegenteil. Es geht um eine den heutigen Anforderungen gerecht werdenden Weiterentwicklung und Neugestaltung der bundesstaatlichen Kompetenzteilung im Sicherheits- und Polizeirecht. Gestützt auf eine Änderung der Bundesverfassung wäre ein Polizeirahmengesetz des Bundes (auch für die Kantone) eine Möglichkeit. Sie führte wie im Strafprozessrecht zu einer einheitlichen materiell-rechtlichen Grundlage, auf die gestützt die kantonalen (und städtischen) Polizeidienste arbeiten. Unverzichtbare Voraussetzung dafür ist indessen auch eine Änderung von Art. 190 BV: Zur Sicherung der Grundrechte müsste die vollständige Verfassungsgerichtsbarkeit auch auf Bundesgesetze anwendbar sein, ${ }^{115}$ wie die angeführten Beispiele belegen.

114 So auch EDÖB, Jahresbericht 2019/20 (Fn.79), S. 29.

115 MOHLER, Grundzüge (Fn. 55), N266ff.; SGK BV-SCHWEIZER/MOHLER, Vorbem. zur Sicherheitsverfassung N $77 \mathrm{ff}$. 